\title{
Abordagem da violência familiar na Estratégia Saúde da Família: Revisão da literatura
}

\author{
Approach to family violence in the Family Health Strategy: Review of literature
}

\author{
a] Psicóloga, mestre em Psicologia \\ pela Universidade Federal de San- \\ ta Catarina (UFSC), Florianópolis, \\ SC - Brasil, e-mail: \\ psi.beatriz@gmail.com \\ ${ }^{[b]}$ Enfermeira, doutora em \\ Filosofia da Enfermagem pela \\ Universidade Federal de Santa \\ Catarina (UFSC) e professora \\ do Departamento de Saúde \\ Pública da mesma instituição, \\ Florianópolis, SC - Brasil, e-mail: \\ elzacoelho@gmail.com
}

Recebido: 30/03/2011 Received: 03/30/2011

Aprovado: 22/03/2012 Approved: 03/22/2012

\author{
Beatriz Schmidf ${ }^{[a]}$, Elza Salema Berger Coelho ${ }^{[b]}$
}

\begin{abstract}
Resumo
Em virtude dos impactos que a violência familiar acarreta no processo de desenvolvimento daqueles que a vivenciam, afetando aspectos psicológicos, físicos e sociais, ela é considerada, atualmente, um importante problema de saúde no Brasil. O objetivo do presente trabalho foi revisar a produção científica brasileira a respeito da abordagem da violência familiar na Atenção Primária à Saúde (APS) e Estratégia Saúde da Família (ESF). Realizou-se levantamento das produções a partir de buscas nas seguintes bases de dados: Scientific Electronic Library Online (SciELO) e Biblioteca Virtual em Saúde (BVS). Foram utilizados descritores preestabelecidos e indexados no processo de busca pelas publicações. Os resultados indicaram que o tipo de violência mais abordado foi a praticada contra a mulher. Constatou-se predomínio de trabalhos de corte transversal, enfoque empírico-pesquisa, sendo entrevistas e questionários as técnicas mais utilizadas. A categoria temática mais prevalente referiu-se à análise das práticas dos profissionais. Pontua-se a necessidade de melhor descrição dos métodos utilizados nos trabalhos, uma vez que alguns estudos avaliados não informaram detalhes relevantes sobre o procedimento metodológico adotado. Indica-se a importância de desenvolvimento de pesquisas que abordem a avaliação, por parte de profissionais e usuários dos serviços de saúde, do trabalho que vem sendo desenvolvido na APS e ESF com relação à violência familiar.
\end{abstract}

Palavras-chave: Violência na família. Atenção primária à saúde. Saúde da família.

\section{Abstract}

Domestic violence is currently considered a major health problem in Brazil. This type of violence affects psychological, physical and social aspects of the victims and produces important impacts on their development process. The aim of this study was to review the Brazilian scientific literature on the approach to domestic violence in the Primary Health Care (APS in Portuguese) and the Family Health Strategy (ESF in Portuguese), that are part of the health care policies of the Brazilian government. A survey of the literature was conducted in the following databases: Scientific Electronic Library Online (SciELO) and the Biblioteca Virtual em Saúde (BVS). Preestablished and indexed descriptors were used in the survey process. The results showed that

Psicol. Argum. 2013 jul./set., 31 (74), 373-381 
the most studied type of violence is the violence against women. It was found the prevalence of cross-sectional studies and empirical-research approach, with interviews and questionnaires being the most used techniques. The prevalent theme category was related to the analysis of the practices of professionals. The article highlights the need for better description of the methods used in these works, since some reviewed studies did not provide relevant details about their methodological process. It is pointed out the importance of developing researches addressing the evaluation of the work that is being developed at the APS and FHS in relation to family violence by both professionals and users of the health care services.

Keywords: Family violence. Primary health care. Family health.

\section{Introdução}

O objetivo do presente trabalho é identificar e caracterizar o que foi produzido na literatura científica brasileira acerca da abordagem da violência familiar na Atenção Primária à Saúde (APS) e Estratégia Saúde da Família (ESF). A violência tem sido crescentemente considerada um problema de saúde pública, nacional e internacionalmente, associando-se a consequências de curto e longo prazo no desenvolvimento psicossocial e na saúde de indivíduos, famílias, comunidades e nações (Lima, Büchele \& Clímaco, 2008; Organização Mundial da Saúde [OMS], 2002). Difundida em todo o tecido social, a violência acontece em espaços privados e públicos, em relações institucionais, grupais e interpessoais (Schraiber, D’Oliveira \& Couto, 2006), estando relacionada a custos econômicos e sociais, tanto para o Estado quanto para as famílias, e à perda de potenciais anos de vida (Ministério da Saúde, 2009).

Dentre os diferentes tipos de violência, destaca-se o que ocorre nos contextos familiares. Reichenheim, Dias e Moraes (2006) consideram a violência familiar como um fenômeno multifacetado que envolve todos os integrantes da família, não estando restrito, portanto, a uma relação ou pessoa específica. Tal qual a violência física, psicológica, sexual ou a negligência, caracteriza-se por diversas modalidades de violência interpessoal, mas neste caso ocorrendo no núcleo familiar (Penna, Santos \& Souza, 2004). A violência familiar atinge o desenvolvimento humano no que tange aos aspectos psicológicos, físicos e sociais, gerando desdobramentos no sistema familiar como um todo e na sociedade à qual esse sistema pertence (Antoni, Barone \& Koller, 2007). Por conta de sua gravidade, é considerada, atualmente, uma importante questão de saúde no Brasil (Lima, 2004).
Diferentes pesquisas têm indicado os impactos na saúde e desenvolvimento de crianças e adolescentes que sofrem violência nos contextos familiares (Antoni et al., 2007; Arpini, Quintana \& Gonçalves, 2010; Lima, 2004; Luna, Ferreira \& Vieira, 2010; Pesce, 2009). Além disso, um grande número de estudos também enfoca a violência contra a mulher praticada nos lares (Audi, Segall-Corrêa, Santiago, Andrade \& PèrezEscamila, 2008; Bonfim, Lopes, \& Peretto, 2010; Borsoi, Brandão \& Cavalcanti, 2009; Deeke, Boing, Oliveira \& Coelho, 2009; Kronbauer \& Meneghel, 2005; Wilhelm \& Tonet, 2007). Contudo, Schraiber et al. (2006) identificaram, a partir de uma revisão crítica da produção científica a respeito da temática da violência e seus impactos sobre a saúde, que nos estudos de violência contra a mulher o tema família foi pouco presente, apesar da utilização dos termos violência doméstica ou intrafamiliar. Reichenheim et al. (2006) constataram a completa ausência de estudos que estimassem a probabilidade de co-ocorrência das diferentes formas de violência familiar, como a violência conjugal e contra filhos, por exemplo, em um trabalho de revisão da literatura nacional.

Penna et al. (2004) apontaram, com base em uma revisão da literatura científica sobre violência doméstica na área da Saúde Pública, que a projeção dessa temática como foco de interesse de pesquisas é bastante recente, sendo ainda escassa a sua produção. Ademais, constataram ênfase em estudos sobre a violência sofrida por mulheres e crianças/ adolescentes no seio familiar, em detrimento de trabalhos que privilegiem idosos, homens e pessoas com deficiência, identificando, portanto, lacunas na produção do conhecimento.

Considerando a violência na família um problema de saúde pública e também o impacto que a mesma acarreta no desenvolvimento humano, nas 
relações interpessoais, comunitárias e na sociedade de forma geral, é fundamental que se pense em ações para abordá-la em serviços de saúde, especialmente pelas equipes de saúde da família. Essas equipes fazem parte da Estratégia de Saúde da Família, implantada em 1994 com o objetivo de reverter o modelo assistencial, visando consolidar os princípios doutrinários do Sistema Único de Saúde por meio da universalidade de acesso, da integralidade na atenção à saúde e da descentralização de planejamento e político-administrativa (Andrade, Barreto \& Bezerra, 2006; Böing, Crepaldi \& Moré, 2009; Ministério da Saúde, 2006).

Compõem as equipes de saúde da família, no mínimo, médico generalista, enfermeiro, auxiliar de enfermagem ou técnico de enfermagem e agentes comunitários de saúde, que trabalham com vistas ao desenvolvimento de ações focalizadas na saúde, direcionadas às famílias em seu contexto, de forma integral, contínua, ativa e descentralizada, com abordagem interdisciplinar e intersetorial. A Estratégia de Saúde da Família constitui-se como "porta de entrada" para o sistema, sendo organizadora do mesmo, encaminhando usuários para os níveis de maior complexidade de atenção, sempre que necessário. Suas ações são voltadas para promoção, prevenção, recuperação, reabilitação e cuidados paliativos, com foco na unidade familiar e ênfase na criação de vínculos, laços de compromisso e corresponsabilidade entre os profissionais e a população por eles atendida (Andrade et al., 2006; Böing et al., 2009; Ministério da Saúde, 2006).

Com base no modelo de processo de trabalho da Estratégia de Saúde da Família, que concebe os usuários em seu contexto na perspectiva da integralidade, percebe-se que as equipes de saúde da família apresentam plenas condições para o enfrentamento da violência familiar (Andrade \& Fonseca, 2008). Considerando, então, o 'local' privilegiado que essas equipes ocupam e os desdobramentos da violência familiar no processo de desenvolvimento psicossocial e na saúde daqueles que a vivenciam, o presente trabalho apresenta uma revisão da produção científica nacional sobre abordagem da violência familiar na Atenção Primária à Saúde (APS) e Estratégia Saúde da Família (ESF).

\section{Método}

O levantamento de dados foi realizado a partir de buscas nas bases de dados Scientific Electronic Library Online (SciELO) e Biblioteca Virtual em Saúde (BVS). Foram analisados artigos escritos em língua portuguesa e publicados em periódicos científicos nacionais com o intuito de conhecer as especificidades dos estudos desenvolvidos sobre a abordagem da violência familiar pela ESF e APS na literatura brasileira. 0 período de publicação dos documentos foi delimitado entre 1994 (ano de implantação da ESF) e 2010.

A seleção dos trabalhos obedeceu a critérios específicos, considerando os termos preestabelecidos localizados nos descritores de assunto, título, resumo e/ou palavras-chave dos estudos encontrados. Os descritores selecionados referiam-se a termos indexados, obtidos a partir de consulta efetuada nos Descritores em Ciências da Saúde da BVS (DeCS) e na Terminologia em Psicologia da BVS-Psicologia. A pesquisa foi realizada pela combinação de dois termos, um deles relacionado à ocorrência de violência em âmbito familiar, e o outro, a serviço de saúde. Recorreu-se ao operador lógico 'AND' para combinação dos descritores e termos utilizados para o rastreamento das publicações. As estratégias de busca foram as seguintes: (1) violência doméstica AND estratégia saúde da família; (2) violência doméstica AND programa saúde da família; (3) violência doméstica AND sistema único de saúde; (4) violência doméstica AND atenção primária à saúde; (5) violência doméstica AND saúde da família; (6) violência na família AND estratégia saúde da família; (7) violência na família AND programa saúde da família; (8) violência na família AND sistema único de saúde; (9) violência na família AND atenção primária à saúde; (10) violência na família AND saúde da família.

Os artigos obtidos foram selecionados e importados para o software End Note X41; nessa etapa, ocorreu a avaliação dos resumos, a retirada daqueles que não estavam relacionados à temática desta pesquisa e a exclusão de trabalhos duplicados (ou seja, disponibilizados em mais de uma base de dados). Em algumas situações, a referência era importada para o software sem a contemplação do resumo. Nessas ocasiões, houve a captação do resumo para inclusão na biblioteca formada no End Note. Quando no resumo não

10 software End Note X4 é um aplicativo capaz de gerenciar referências bibliográficas, importando-as diretamente de bancos de dados da Web, para criação de uma biblioteca pessoal. Número da licença para uso do aplicativo: 5071401966.

Psicol. Argum. 2013 jul./set., 31 (74), 373-381 
esclarecia suficientemente se o estudo se referia, de fato, à abordagem da violência familiar pelos profissionais de saúde da família, o texto era consultado na íntegra, sempre que disponível.

Os artigos cujos resumos foram avaliados como concernentes à abordagem da violência familiar pela APS e ESF foram lidos integralmente, quando disponíveis, com o objetivo de elaborar um quadro de referências com os seguintes dados: autor(es); ano de publicação; periódico onde foi publicado; enfoque do estudo: teórico (incluindo aqui artigos de opinião, revisão de literatura e documental), empírico-pesquisa e intervenção prática; tipo de estudo (transversal ou longitudinal); técnicas utilizadas (observações, entrevistas, questionários/escalas, testes, pesquisa bibliográfica, grupo operativo, combinado ou não se aplica); análise de dados (qualitativa ou quantitativa); tipos de violência abordados e objetivos do estudo. A elaboração desse quadro de referências foi efetuada utilizando o software Excel. A análise qualitativa ocorreu com base na técnica de análise temática, que consiste em identificar os núcleos de sentido da comunicação (Bardin, 1977).

\section{Resultados e discussão}

Foram identificados, inicialmente, 575 artigos encontrados a partir dos descritores preestabelecidos nas bases de dados pesquisadas. Dos artigos obtidos, 455 encontravam-se na BVS e 120 no SciELO. Com a manipulação para exclusão das duplicatas, restaram 183 trabalhos que tiveram seus resumos lidos e avaliados, visando verificar se tratavam, de fato, da abordagem da violência ocorrida em âmbito familiar pela Atenção Primária à Saúde e Estratégia Saúde da Família. Dos 183 resumos analisados, identificou-se que 19 versavam sobre o tema objeto do presente estudo.

Ocorreu a leitura integral de 15 desses 19 trabalhos. Quatro trabalhos não foram lidos integralmente porque o texto completo não estava disponível. Nesses casos, onde não foi possível o acesso ao texto completo, o resumo serviu como fonte exclusiva de análise e obtenção de dados referentes aos estudos desenvolvidos.

A partir dos trabalhos selecionados, pôde-se identificar que a publicação do primeiro artigo acerca da temática ocorreu em 1999, cinco anos após o surgimento da ESF como política nacional de saúde.
Nota-se, entretanto, uma maior produção científica, sobretudo nos últimos cinco anos, uma vez que 13 dos 19 artigos foram publicados no decorrer desse período, o que representa $68,4 \%$ dos estudos analisados (Tabela 1).

Tabela 1 - Número de artigos segundo ano de publicação

\begin{tabular}{lcc}
\hline Variável & $\mathbf{n}$ & \% \\
\hline Ano de publicação & & \\
1999 & 1 & 5,3 \\
2000 & 1 & 5,3 \\
2002 & 1 & 5,3 \\
2005 & 2 & 10,5 \\
2006 & 1 & 5,3 \\
2007 & 4 & 21,1 \\
2008 & 5 & 26,3 \\
2009 & 2 & 10,5 \\
2010 & 2 & 10,5 \\
Total & $\mathbf{1 9}$ & $\mathbf{1 0 0}$ \\
\hline
\end{tabular}

Fonte: Dados da pesquisa.

O periódico com maior número de publicações concernentes à abordagem da violência familiar pela APS e ESF foi a Revista de Saúde Pública, com quatro artigos, seguido da Ciência \& Saúde Coletiva e da Revista da Escola de Enfermagem da USP, com três e dois artigos, respectivamente (Tabela 2). Esses três periódicos científicos foram responsáveis pela publicação de nove dos 19 artigos avaliados, o que representa quase a metade da produção referente ao tema $-47,4 \%$, mais precisamente.

Os tipos de violência abordados nos artigos analisados foram: contra a mulher (13 estudos, 68,4\%); contra idosos (quatro estudos, 21,1\%); e contra crianças e/ou adolescentes (dois estudos, 10,5\%) (Tabela 3). É notória maior prevalência de estudos relativos à violência contra a mulher praticada nas famílias. Esse resultado confirma o obtido por Penna et al. (2004), que encontraram em um trabalho de revisão da literatura sobre violência doméstica na área da saúde pública, cuja amostra total era constituída por 29 artigos, 15 que focalizavam mulheres e 11 crianças e/ou adolescentes. É possível considerar, portanto, a necessidade de pesquisas sobre a violência familiar vivenciada, por exemplo, por homens e pessoas com deficiência. 
Tabela 2 - Periódicos publicados

\begin{tabular}{lcc}
\hline Periódicos Publicados & n & \% \\
\hline Revista de Saúde Pública & 4 & 21,05 \\
Ciência \& Saúde Coletiva & 3 & 15,79 \\
Revista da Escola de Enfermagem da USP & 2 & 10,53 \\
Acta Médica (Porto Alegre) & 1 & 5,26 \\
Cadernos de Saúde Pública & 1 & 5,26 \\
Ciência, Cuidado e Saúde & 1 & 5,26 \\
Divulgação em Saúde para Debate & 1 & 5,26 \\
Escola Anna Nery Revista de Enfermagem & 1 & 5,26 \\
Interface - Comunicação, Saúde, Educação & 1 & 5,26 \\
Psicologia, Ciência e Profissão & 1 & 5,26 \\
Revista de Medicina (São Paulo) & 1 & 5,26 \\
Revista Eletrônica de Enfermagem & 1 & 5,26 \\
Saúde e Sociedade & 1 & 5,26 \\
Total & $\mathbf{1 9}$ & $\mathbf{1 0 0}$ \\
\hline
\end{tabular}

Fonte: Dados da pesquisa.

Percebeu-se também a inexistência de estudos que estimem a ocorrência de violência entre diferentes membros da família (como ocorrência simultânea de violência conjugal e contra filhos, ou violência contra filhos e entre irmãos em uma determinada família). Tampouco ocorreram estudos acerca da transmissão intergeracional da violência, isto é, sobre a forma que a violência se propaga entre gerações.

Além disso, no que diz respeito ao tipo de estudo, nota-se a predominância de trabalhos de corte transversal, ou seja, aqueles que focalizam um momento específico da vida do(s) participante(s). Os 14 estudos em que foi possível identificar o corte eram transversais. Em três trabalhos em que apenas o resumo estava disponível, não houve possibilidade de avaliar a estratégia de corte utilizada. Além desses três artigos, em outros dois não cabia analisar o corte, já que se tratavam de artigos teóricos (Tabela 3). Sendo assim, não foi identificado corte longitudinal em nenhum dos estudos analisados. Os estudos longitudinais se caracterizam pela coleta de dados ao longo do tempo, permitindo inferências sobre mudanças, determinantes e efeitos (Sampieri, Collado \& Lucio, 2006), o que forneceria resultados interessantes para analisar os impactos da violência familiar no decorrer de um determinado período.

No que tange ao enfoque dos estudos, 11 foram empíricos-pesquisas $(57,9 \%)$, três foram teóricos
$(15,8 \%)$, um era intervenção prática $(5,3 \%)$ e quatro não puderam ser identificados. Nota-se a prevalência de pesquisas empíricas sobre os demais enfoques, como pode ser observado na Tabela 3.

As técnicas mais utilizadas foram as entrevistas, presentes em cinco trabalhos $(26,3 \%)$ e os questionários, também encontrados em cinco artigos $(26,3 \%)$. Um trabalho utilizou exclusivamente a observação (5,3\%), e outro trabalho empregou observação associada a entrevista (5,3\%) (Tabela 3).

A análise de dados foi qualitativa em oito trabalhos $(42,1 \%)$ e quantitativa em sete $(36,8 \%)$, não podendo ser identificada em quatro deles (Tabela 3).

Tabela 3 - Tipos de violência abordados, tipos de estudo, enfoque dos estudos, análise de dados

\begin{tabular}{lcc}
\hline Variável & $\mathbf{n}$ & $\mathbf{\%}$ \\
\hline Tipos de violência abordados & 13 & 68,4 \\
Violência contra mulheres & 4 & 21,1 \\
Violência contra idosos & 2 & 10,5 \\
Violência contra crianças e adolescentes & $\mathbf{1 9}$ & $\mathbf{1 0 0}$ \\
Total & $\mathbf{n}$ & $\mathbf{\%}$ \\
\hline Tipo de estudo & 14 & 73,7 \\
\hline Transversal & 5 & 26,3 \\
Não identificado & $\mathbf{1 9}$ & $\mathbf{1 0 0}$ \\
Total & $\mathbf{n}$ & $\mathbf{9}$ \\
\hline Enfoque dos estudos & 11 & 57,9 \\
\hline Empírico-pesquisa & 3 & 15,8 \\
Teórico & 1 & 5,3 \\
Intervenção prática & 4 & 21,1 \\
Não identificado & $\mathbf{1 9}$ & $\mathbf{1 0 0}$ \\
Total & $\mathbf{n}$ & $\mathbf{9}$ \\
\hline Técnicas utilizadas & 5 & 26,3 \\
\hline Entrevista & 5 & 26,3 \\
Questionário & 1 & 5,3 \\
Observação & 1 & 5,3 \\
Combinado (observação e entrevista) & 7 & 36,8 \\
Não identificado & $\mathbf{1 9}$ & $\mathbf{1 0 0}$ \\
\hline Total & $\mathbf{n}$ & $\mathbf{9 0}$ \\
\hline Análise de dados & 8 & 42,1 \\
\hline Qualitativa & 7 & 36,8 \\
Quantitativa & 4 & 21,1 \\
Não identificado & $\mathbf{1 9}$ & $\mathbf{1 0 0}$ \\
Total & & \\
\hline & & \\
\hline
\end{tabular}

Fonte: Dados da pesquisa.

Psicol. Argum. 2013 jul./set., 31 (74), 373-381 
Essa dificuldade de identificação, tanto da estratégia adotada para análise de dados, quanto do tipo de estudo, enfoque e técnicas utilizadas, pode ter relação com as defasagens na descrição do método ou, até mesmo, falta de rigor metodológico no processo de elaboração do trabalho. Considerando a importância de uma descrição minuciosa do método, visando a replicabilidade do trabalho - o que se relaciona com a possibilidade de avanço na produção do conhecimento científico - , seria importante que os autores se atentassem para a elaboração dessa seção nos artigos publicados, com o objetivo de facilitar a comunicação científica.

Os objetivos dos trabalhos avaliados foram diversos, conforme pode se observar no Quadro 1.

Considerando que os artigos avaliados tiveram objetivos diversos, as autoras realizaram uma

Quadro 1 - Objetivos dos trabalhos avaliados, seus autores e ano de publicação

\begin{tabular}{|c|c|}
\hline Autor e ano de publicação & Objetivo \\
\hline Cavalcanti (1999) & $\begin{array}{l}\text { Apresentar as observações e dados de um estudo exploratório sobre as percepções e vivências de } \\
\text { profissionais do Programa Médico de Família de Niterói em contato com situações de violência } \\
\text { doméstica contra crianças e adolescentes. }\end{array}$ \\
\hline $\begin{array}{l}\text { Schraiber, D’Oliveira, França } \\
\text { Junior, Strake e Oliveira } \\
(2000)\end{array}$ & $\begin{array}{l}\text { Compreender a situação referente à capacidade que apresentam os serviços para acolher e } \\
\text { responder a demandas de mulheres em situação de violência, em termos das possibilidades já } \\
\text { existentes na rotina usual de atenção à saúde da mulher e das limitações desse serviço. }\end{array}$ \\
\hline $\begin{array}{l}\text { Schraiber, D’Oliveira, Fran- } \\
\text { ça-Junior e Pinho (2002) }\end{array}$ & $\begin{array}{l}\text { Investigar casos de violência contra a mulher, identificando a natureza do ato perpetrado, a quali- } \\
\text { dade/gravidade da violência e a relação do(a) agressor(a) com a mulher. }\end{array}$ \\
\hline $\begin{array}{l}\text { Kronbauer e Meneghel } \\
\text { (2005) }\end{array}$ & $\begin{array}{l}\text { Dimensionar e caracterizar o perfil da violência de gênero perpetrada por companheiro, abor- } \\
\text { dando as situações de violência física, psicológica e sexual que resultaram ou podem resultar em } \\
\text { dano à vida e ou à integridade da mulher. }\end{array}$ \\
\hline $\begin{array}{l}\text { Pedreira, Biondo, Kotovicz, } \\
\text { Souza e Schraider (2005) }\end{array}$ & $\begin{array}{l}\text { Estudar a prevalência de violência doméstica física e/ou sexual contra a mulher e suas repercus- } \\
\text { sões para a saúde. }\end{array}$ \\
\hline $\begin{array}{l}\text { Marinheiro, Vieira e Souza } \\
(2006)\end{array}$ & $\begin{array}{l}\text { Determinar a prevalência da violência contra a mulher cometida por parceiro íntimo entre usuá- } \\
\text { rias de um centro de saúde distrital em Ribeirão Preto. }\end{array}$ \\
\hline $\begin{array}{l}\text { Lazarotto, Dias, Santos, e } \\
\text { Gauer (2007) }\end{array}$ & $\begin{array}{l}\text { Abordar o tema dos maus-tratos aos idosos e a importância do reconhecimento e da atuação dos } \\
\text { profissionais da saúde no que se refere a esse problema. }\end{array}$ \\
\hline $\begin{array}{l}\text { Meira, Gonçalves e Xavier } \\
\text { (2007) }\end{array}$ & $\begin{array}{l}\text { Identificar fatores de risco como preditores de violência intrafamiliar contra o idoso com base em } \\
\text { história oral dos familiares cuidadores sobre sua relação de cuidado com o parente idoso. }\end{array}$ \\
\hline Oliveira e Fonseca (2007) & $\begin{array}{l}\text { Analisar as práticas de profissionais que atuam no Programa de Saúde da Família no estado de } \\
\text { Minas Gerais voltado para mulheres em situação de violência sexual. }\end{array}$ \\
\hline $\begin{array}{l}\text { Scaranto, Biazevic e Mi- } \\
\text { chel-Crosato (2007) }\end{array}$ & $\begin{array}{l}\text { Investigar a percepção dos agentes comunitários de saúde sobre a violência doméstica contra a } \\
\text { mulher. }\end{array}$ \\
\hline Andrade e Fonseca (2008) & $\begin{array}{l}\text { Oportunizar reflexão sobre a possibilidade de enfrentamento da violência doméstica a partir do } \\
\text { trabalho das equipes de saúde da família. }\end{array}$ \\
\hline Audi et al. (2008) & $\begin{array}{l}\text { Identificar fatores associados à violência doméstica, caracterizada pela violência psicológica e } \\
\text { física e/ou sexual, perpetrada } \\
\text { por parceiro íntimo durante o período gestacional. }\end{array}$ \\
\hline $\begin{array}{l}\text { Moraes, Apratto Júnior e } \\
\text { Reichenheim (2008) }\end{array}$ & $\begin{array}{l}\text { Estimar a prevalência da violência física contra idosos no ambiente doméstico em população } \\
\text { adscrita ao Programa Médico de Família } \\
\text { de Niterói. }\end{array}$ \\
\hline $\begin{array}{l}\text { Silva, Oliveira, Joventino e } \\
\text { Moraes (2008) }\end{array}$ & $\begin{array}{l}\text { Conhecer as percepções do idoso sobre violência; identificar quais os tipos de violência que o } \\
\text { afetam; detectar como ele reage frente a } \\
\text { um ato que considera violência; relatar se há diferença de reação dependendo da pessoa agente } \\
\text { da violência/agressão; descobrir a quem ele recorre quando se sente agredido. }\end{array}$ \\
\hline $\begin{array}{l}\text { Schraiber e D’Oliveira } \\
\text { (2008) }\end{array}$ & $\begin{array}{l}\text { Discutir, com base em pesquisas e experiências em serviços de saúde, contribuições para lidar } \\
\text { com a violência contra a mulher, do diagnóstico dos casos até a proposta de rede inter-setorial. }\end{array}$ \\
\hline Borsoi et al. (2009) & $\begin{array}{l}\text { Conhecer como os serviços pesquisados absorvem e encaminham as demandas relativas às situa- } \\
\text { ções de violência percebidas pelos profissionais de saúde no atendimento cotidiano às usuárias. }\end{array}$ \\
\hline $\begin{array}{l}\text { D’Oliveira, Schraiber, Hana- } \\
\text { da e Durand (2009) }\end{array}$ & $\begin{array}{l}\text { Tratar das possibilidades de atuação do campo da saúde na abordagem da violência contra a } \\
\text { mulher desde suas práticas assistenciais nos serviços e baseadas na perspectiva de gênero. }\end{array}$ \\
\hline
\end{tabular}


Quadro 1 - Objetivos dos trabalhos avaliados, seus autores e ano de publicação

\begin{tabular}{ll}
\hline Autor e ano de publicação & Objetivo \\
\hline Bonfim et al. (2010) & $\begin{array}{l}\text { Identificar e analisar condutas e estratégias utilizadas por profissionais de saúde durante o pré- } \\
\text {-natal, na suspeita de casos e na violência declarada, e discutir a problemática dos atendimentos } \\
\text { às gestantes em situação de violência. }\end{array}$ \\
\hline Luna et al. (2010) & $\begin{array}{l}\text { Analisar o processo de notificação de maus-tratos em crianças e adolescentes por médicos, enfer- } \\
\text { meiros e cirurgiões-dentistas da Estratégia Saúde da Família em Fortaleza. }\end{array}$ \\
\hline
\end{tabular}

Fonte: Dados da pesquisa.

análise qualitativa, com base na análise temática proposta por Bardin (1977), na qual foram evidenciadas seis unidades temáticas, a saber: (1) Análise das práticas dos profissionais; (2) Capacidade de acolhimento de demandas; (3) Caracterização dos casos de violência familiar; (4) Prevalência; (5) Percepções de profissionais sobre o fenômeno; e (6) Identificação de fatores de risco para a ocorrência de situações de violência familiar.

A categoria temática mais prevalente foi a Análise das práticas dos profissionais, identificada em cinco trabalhos, seguida da Capacidade de acolhimento de demandas e Caracterização dos casos de violência familiar, cada qual presente em quatro estudos. Na sequência vieram as categorias Prevalência e Percepções de profissionais sobre o fenômeno, constatadas em três e dois artigos, respectivamente. Um trabalho versou sobre a Identificação de fatores de risco para a ocorrência de situações de violência familiar.

\section{Considerações finais}

Esre trabalho apresentou um panorama geral acerca das pesquisas que vêm sendo desenvolvidas sobre a abordagem da violência familiar pela APS e ESF, levantando algumas características importantes dessas produções. Destacou-se a prevalência de estudos realizados com mulheres vítimas de violência familiar e a inexistência de pesquisas sobre outros membros da família, como homens e pessoas com deficiência. Ademais, também não foram identificados estudos longitudinais, importantes para a avaliação do impacto da violência ao longo do tempo.

Outra característica da amostra foi a inexistência de pesquisas que avaliassem o trabalho desenvolvido pela APS e pela ESF com relação à violência familiar. Estudos de avaliação dos efeitos das ações em curto, médio e longo prazo, satisfação com os atendimentos prestados ou programas desenvolvidos, por exemplo, não foram identificados. Nota-se aí uma lacuna no conhecimento produzido até o presente momento acerca da temática do presente trabalho.

Com relação ao enfoque dos estudos, houve paridade quanto à abordagem qualitativa e quantitativa dos dados; contudo, não se observaram análises qualitativas e quantitativas utilizadas conjuntamente na amostra, o que poderia ser implementado a partir de novas pesquisas, considerando que não são excludentes. São, na verdade, complementares, podendo ser utilizadas concomitantemente a fim de reduzir possíveis limitações de cada uma delas (Fleith \& Costa Junior, 2005). Essa junção de diferentes abordagens de dados é importante para o estudo de fenômenos complexos, como é o caso da violência familiar.

Notou-se rande número de estudos empíricos, em detrimento de intervenções práticas, como estudos de caso, e estudos teóricos, como revisões de literatura. Nesse sentido, o presente artigo é relevante, haja vista que pretende preencher uma lacuna no conhecimento científico disponível.

Enfatiza-se, portanto, a importância de mais produções científicas sobre a violência que ocorre nos contextos familiares, cuja projeção como foco de interesse de pesquisas ainda é limitada (Penna, 2004). Os resultados dessas pesquisas poderiam contribuir para a melhoria da atenção prestada à clientela, constituindo-se como importante meio para informação de profissionais, bem como subsídio para a preparação de programas de formação continuada dos mesmos.

Psicol. Argum. 2013 jul./set., 31 (74), 373-381 


\section{Referências}

Andrade, L. O. M., Barreto, I. C. H. C., \& Bezerra, R. C. (2006). Atenção primária à saúde e a estratégia saúde da família. In G. W. Campos, M. C. S. Minayo, M. Akerman, M. Drumond Júnior \& Y. M. Carvalho (Org.). Tratado de saúde coletiva (pp. 783-838). São Paulo: Hucitec; Rio de Janeiro: Fiocruz.

Andrade, C. J. M., \& Fonseca, R. M. G. S. (2008). Considerações sobre violência doméstica, gênero e o trabalho das equipes de saúde da família. Revista da Escola de Enfermagem da USP, 42(3), 591-595.

Antoni, C., Barone, L. R., \& Koller, S. H. (2007). Indicadores de risco e proteção em famílias fisicamente abusivas. Psicologia: Teoria e Pesquisa, 23(2), 125-132.

Arpini, D. M., Quintana, M. A. Gonçalves, C. S. (2010). Relações familiares e violência em adolescentes em situação de rua. Psicologia Argumento, 28(63), 325-336.

Audi, C. A. F., Segall-Corrêa, A. M., Santiago, S. M., Andrade, M. G. G., \& Pèrez-Escamila, R. (2008). Violência doméstica na gravidez: Prevalência e fatores associados. Revista de Saúde Pública, 42(5), 877-885.

Bardin, L. (1977). Análise de Conteúdo. Lisboa: Edições 70.

Böing, E., Crepaldi, M. A., \& Moré, C. L. O. O. (2009). A epistemologia sistêmica como substrato à atuação do psicólogo na atenção básica. Psicologia: Ciência e Profissão, 29(4), 828-845.

Bonfim, E. G., Lopes, M. J. M., \& Peretto, M. (2010). Os registros profissionais do atendimento pré-natal e a (in)visibilidade da violência doméstica contra a mulher. Escola Anna Nery - Revista de Enfermagem, 14(1), 97-104.

Borsoi, T. S., Brandão, E. R., \& Cavalcanti, M. L. T. (2009). Ações para o enfrentamento da violência contra a mulher em duas unidades de atenção primária à saúde no município do Rio de Janeiro. Interface Comunicação, Saúde, Educação, 13(28), 165-174.

Cavalcanti, M. L. T. (1999). Prevenção da violência doméstica na perspectiva dos profissionais de saúde da família. Ciência e Saúde Coletiva, 4(n. esp.), 193-200.

Deeke, L. P., Boing, A. F., Oliveira, W. F., \& Coelho, E. B. S. (2009). A dinâmica da violência doméstica: Uma análise a partir dos discursos da mulher agredida e de seu parceiro. Saúde e Sociedade, 18(2), 248-258.
D’Oliveira, A. F. P. L., Schraiber, L. B., Hanada, H., \& Durand, J. (2009). Atenção integral à saúde de mulheres em situação de violência de gênero: Uma alternativa para a atenção primária em saúde. Ciência e Saúde Coletiva, 14(4), 1037-1050.

Fleith, D. S., \& Costa Junior, A. L. (2005). Métodos de pesquisa em psicologia: 0 que é relevante considerar? In M. A. Dessen \& A. L. Costa Junior (Org.). A ciência do desenvolvimento humano: Tendências atuais e perspectivas futuras (pp. 37-49). Porto Alegre: Artmed.

Kronbauer, J. F. D., \& Meneghel, S. N. (2005). Perfil da violência de gênero perpetrada por companheiro. Revista de Saúde Pública, 39(5), 695-701.

Lazarotto, F. D. P., Dias, A. C., Santos, E. L. M., \& Gauer, G. J. C. (2007). Maus-tratos aos idosos. Acta Médica (Porto Alegre), 28, 409-419.

Lima, C. A. (Coord.) (2004). Violência faz mal à saúde. Brasília: Ministério da Saúde.

Lima, D. C., Büchele, F., \& Clímaco, D. A. (2008). Homens, gênero e violência contra a mulher. Saúde e Sociedade, 17(2), 69-81.

Luna, G. L. M., Ferreira, R. C., \& Vieira, L. J. E. S. (2010). Notificação de maus-tratos em crianças e adolescentes por profissionais da Equipe Saúde da Família. Ciência e Saúde Coletiva, 15(2), 481-491.

Marinheiro, A. L. V., Vieira, E. M., \& Souza, L. (2006). Prevalência da violência contra a mulher usuária de serviço de saúde. Revista de Saúde Pública, 40(4), 604-610.

Meira, E. C., Gonçalves, L. H. T., \& Xavier, J. O. (2007). Relatos orais de cuidadores de idosos doentes e fragilizados acerca dos fatores de risco para violência intrafamiliar. Ciência, Cuidado e Saúde, 6(2), 171-180.

Ministério da Saúde (2006). Portaria n. 648 de 28 de março de 2006. Aprova a Política Nacional de Atenção Básica, estabelecendo a revisão de diretrizes e normas para a organização da atenção básica para o Programa Saúde da Família (PSF) e o Programa Agentes Comunitários de Saúde (PACS). Recuperado em 11 set. 2013, de http://bvsms.saude.gov.br/bvs/ saudelegis/gm/2006/prt0648_28_03_2006.html

Ministério da Saúde (2009). Impacto da violência na saúde das crianças e adolescentes. Brasília: Editora MS. Recuperado em 22 mar. 2011, de http://portal. saude.gov.br/portal/arquivos/pdf/cartilha_impacto_violencia_final.pdf 
Moraes, C. L., Apratto Júnior, P. C., \& Reichenheim, M. E. (2008). Rompendo o silêncio e suas barreiras: Um inquérito domiciliar sobre a violência doméstica contra idosos em área de abrangência do Programa Médico de Família de Niterói, Rio de Janeiro, Brasil. Cadernos de Saúde Pública, 24(10), 2289-2300.

Oliveira, C. C., \& Fonseca, R. M. G. S. (2007). Práticas dos profissionais das equipes de saúde da família voltadas para as mulheres em situação de violência sexual. Revista Escola de Enfermagem USP, 41(4), 605-612.

Organização Mundial de Saúde [OMS] (2002). World report on violence and health. Recuperado em 10 mar. 2011, de http://whqlibdoc.who.int/publications/2002/9241545615_eng.pdf

Pedreira, F. A., Biondo, M., Kotovicz, F., Souza, F. A. J., \& Schraiber, L. B. (2005). Violência doméstica e saúde da mulher. Revista de Medicina, 84(2), 45-54.

Penna, L. H. G., Santos, N. C., \& Souza, E. R. (2004). A produção científica sobre violência doméstica na área da saúde pública. Revista de Enfermagem UERJ, 12 (n. esp.), 192-198.

Pesce, R. (2009). Violência familiar e comportamento agressivo e transgressor na infância: Uma revisão da literatura. Ciência e Saúde Coletiva, 14(2), 507-518.

Reichenheim, M. E., Dias, A. S., \& Moraes, C. L. (2006). Co-ocorrência de violência física conjugal e contra filhos em serviços de saúde. Revista de Saúde Pública, 40(4), 595-603.

Sampieri, R. H., Collado, C. F., \& Lucio, P. B. (2006). Metodologia de pesquisa. Porto Alegre: McGraw Hill.
Scaranto, C. A. A., Biazevic, M. G. H, \& Michel-Crosato, E. (2007). Percepção dos agentes comunitários de saúde sobre a violência doméstica contra a mulher. Psicologia Ciência e Profissão, 27(2), 694-705.

Schraiber, L. B., D’Oliveira, A. F. P. L., \& Couto, M. T. (2006). Violência e saúde: Estudos científicos recentes. Revista de Saúde Pública, 40(n. esp.), 112-120.

Schraiber, L. B., D’Oliveira, A. F. P. L., França Junior, I., Strake, S. S., \& Oliveira, E. A. (2000). A violência contra mulheres: Demandas espontâneas e busca ativa em unidade básica de saúde. Saúde e Sociedade, 9(1-2), 3-15.

Schraiber, L. B., D’Oliveira, A. F. P. L., França Junior, I., \& Pinho, A. A. (2002). Violência contra a mulher: Estudo em uma unidade de atenção primária à saúde. Revista de Saúde Pública, 36(4), 470-477.

Schraiber, L. B., \& D’Oliveira, A. F. P. L. (2008). Violência contra mulheres e promoção dos direitos humanos: A contribuição do campo da Saúde. Divulgação em Saúde para Debate, 41, 47-55.

Silva, M. J., Oliveira, T. M., Joventino, E. S, \& Moraes, G. L. A. (2008). A violência na vida cotidiana do idoso: Um olhar de quem a vivencia. Revista Eletrônica de Enfermagem, 10(1), 124-136.

Wilhelm, F. A., \& Tonet, J. (2007). Percepção sobre a violência doméstica na perspectiva de mulheres vitimadas. Psicologia Argumento, 25(51), 401-412.

Psicol. Argum. 2013 jul./set., 31 (74), 373-381 